\title{
Vessel associated adhesion molecules in normal skin and acute graft-versus-host disease
}

\author{
J Norton, J P Sloane, N Al-Saffar, D O Haskard
}

\begin{abstract}
Immunohistological staining of skin from normal donors and bone marrsw transplant recipients was undertaken using antibodies to two vessel associated adhesion molecules, endothelial leucocyte adhesion molecule-1 (ELAM1) and vascular cell adhesion molecule-1 (VCAM-1). In normal skin ELAM-1 staining was restricted to a variable but generally small number of endothelial cells which were significantly increased in graft-versus-host disease (GvHD), but only when the fully developed histological picture of epidermal basal damage and leucocytic infiltration was present. All other biopsy specimens from marrow recipients taken before or after transplantation were similar to those of normal controls even in the presence of a clinical rash consistent with early GvHD. Although VCAM-1 positivity was seen on a few endothelial cells in normal skin, staining was mainly observed on dermal dendritic cells surrounding blood vessels and adnexal structures. In specimens with histological evidence of GvHD, positive perivascular dendritic cells were increased and were accompanied by the appearance of large numbers of similar cells dispersed throughout the upper dermis. Biopsy specimens from marrow recipients before and after transplantation resembled those from normal donors except for the presence of a rash after transplantation when some specimens, which lacked the leucocytic infiltrate diagnostic of GvHD, showed an increase in VCAM-1 positive cells, particularly in the upper dermis.
\end{abstract}

The identification of these cells may therefore be useful in diagnosing early GvHD.

Acute cutaneous graft-versus-host disease (GvHD) is characterised by lymphocytic infiltration of the upper dermis and lower epidermis, and epidermal basal keratinocyte vacuolation, and necrosis. ${ }^{1}$ Similar epidermal changes may be produced by cytotoxic drugs and irradiation given before transplantation, ${ }^{2}$ and are insufficient evidence on which to base a histological diagnosis of GvHD in the absence of a mononuclear cell infiltrate. ${ }^{3}$ In previous studies undertaken in our laboratory we found that biopsy specimens from many patients with rashes who had or subsequently developed strong clinical evidence of GvHD lacked the diagnostic leucocytic infiltrate, especially if taken soon after the onset of the rash. ${ }^{3}$ In some patients with these "negative" specimens HLA-DR expression was increased on epidermal keratinocytes and was strongly associated with the subsequent development of systemic GvHD. ${ }^{4}$

As cultured keratinocytes can be stimulated to synthesise HLA-DR antigens by the addition of cytokines, we suggested that HLA-DR expression on keratinocytes in early GvHD may follow cytokine release in the dermis, consequent on the interaction between recipient dermal cells (possibly in the vascular complex) and incoming donor lymphocytes.

In keratinocyte cultures stimulated this way, HLA-DR expression is preceded by synthesis of the intercellular adhesion molecule ICAM-1 (CD54), ${ }^{5}$ and in a recent histological study we showed increased ICAM-1 staining of keratinocytes in GvHD. ${ }^{6}$ Although there was a strong positive correlation with HLADR positivity, we could find no evidence of increases in biopsy specimens without detectable leucocytic infiltration.

In this study we investigated the expression of two blood vessel associated leucocyte adhesion molecules, endothelial leucocyte adhesion molecule-1 (ELAM-1) ${ }^{7}$ and vascular cell adhesion molecule-1 (VCAM-1) ${ }^{8}$ in the skin of allogeneic marrow recipients to elucidate the possible role for adhesion molecules in acute GvHD and to investigate their possible usefulness in the early diagnosis of this condition.

\section{Methods}

Twenty five $3 \mathrm{~mm}$ punch biopsy specimens of skin were obtained from 24 allogeneic bone marrow recipients ( 15 males and nine females) between days 7 and 43 after transplantation. The underlying disease was acute myeloid leukaemia in 13 cases, chronic granulocytic leukaemia in five, acute lymphoblastic leukaemia in three, aplastic anaemia in one, myelodysplasia in one and myelofibrosis in one. The mean age at transplantation was 28 years (range 13-44). Before transplantation conditioning regimens included combinations of total body irradiation, melphalan, cyclophosphamide and busulphan according to the primary diagnosis. All patients received cyclosporin A alone or cyclosporin A plus methotrexate as prophylaxis against GvHD.

Fifteen biopsy specimens were from patients 
who had a macular erythematous rash mainly affecting the palms, soles, malar area and trunk, but occasionally this was more widespread affecting the back and limbs. Specimens were taken from these sites.

Two groups were selected according to their histological and immunohistological appearances: (1) those specimens exhibiting unequivocal histological evidence of GvHD with leucocytic infiltration of the upper dermis and lower epidermis; and (2) biopsy specimens lacking a leucocytic infiltrate but exhibiting keratinocyte HLA-DR positivity. Ten specimens were from patients with no evidence of a rash at similar times after transplantation.

Control biopsy specimens were obtained from five normal bone marrow donors (three men and two women) of mean age 35 years (range 29-41), from the posterior iliac crest at the time of bone marrow harvest and also from five patients (three men and two women) before transplantation from the site of a Hickman line insertion. Informed consent was obtained.

The skin biopsy specimens were immediately divided in two. One half was snap frozen in liquid nitrogen, the other formalin fixed and paraffin wax embedded for histological examination.

Cryostat sections of $5 \mu \mathrm{m}$ were fixed in formol calcium and chloroform acetone and immunolabelled by the alkaline phosphataseantialkaline phosphatase (APAAP) method using the following monoclonal antibodies: 1.2B6 against ELAM-1; 1.4C3 against VCAM-1, both of IgG1 isotype, ${ }^{9}$ and AMDRPA-1 (Leuk 19), IgG2b isotype, against human monocyte/macrophages (Silenus, Australia through Laboratory Impex Ltd, Middlesex). Polyclonal rabbit antibodies to CD3 and factor VIII related antigen and the monoclonal antibody to monocyte/macrophages EBM11 (CD68) were obtained from Dako Ltd, and HLA-DR antibody L243 from Becton-Dickinson.

The number of ELAM-1 positive vascular endothelial cells was counted and expressed as cells per mm length of epidermis. VCAM-1 positive cells in the dermal vascular complex and free within the upper dermis were counted separately and also expressed as cells per mm length of epidermis. Epidermal lengths were measured using a Leitz Orthoplan Drawing Tube attached to a Laborlux microscope to project an image on to a piece of paper. The tracing of the contour of the granular layer was measured using an opisometer.

Histological appearances fell into three categories $^{3}$ : (1) GvHD, where there was epidermal basal damage associated with leucocytic infiltration of the upper dermis and lower epidermis; (2) epidermal basal abnormalities when there was evidence of epidermal basal damage but no lymphocytic infiltrate; and (3) normal.

In several cases of normal skin and GvHD immunofluorescence studies were undertaken.

Cryostat sections $(5 \mu \mathrm{m})$ were fixed in acetone for 10 minutes. Sections were incubated with the antibodies or the conjugates for 30 minutes to one hour at room tempera- ture. Phosphate buffered saline $0 \cdot 1 \mathrm{M}(\mathrm{pH} 7 \cdot 4)$ was used for the antibody dilutions and washings.

For single labelling with the mouse monoclonal antibodies, sections were incubated with $1.2 \mathrm{~B} 6$ or $1.4 \mathrm{C} 3$ diluted 1 in 100 followed by biotinylated horse anti-mouse IgG (1 in 100) (Vector Laboratories, Peterborough) and finally with streptavidin fluorescene ( 1 in 50). Rabbit anti-CD3 (1 in 60) and anti-factor VIII related antigen ( 1 in 100) were followed by fluorescein-conjugated donkey anti-rabbit Ig ( 1 in 50 ).

Double labelling was performed by incubating the tissue sections with a combination of $1.4 \mathrm{C} 3$ and either anti-CD 3 or anti-factor VIII related antigen at their optimum dilutions, followed by biotinylated horse anti-mouse IgG, and finally with a mixture of streptavidin Texas red ( 1 in 100) and fluorescein isothiocyanate (FITC) conjugated donkey anti-rabbit ( 1 in 50 ) (both conjugates from Amersham International, Buckinghamshire).

For the double labelling with the two mouse monoclonal antibodies, $1.4 \mathrm{C} 3$ and AMDRPA-1, sections were incubated with both antibodies at their optimal dilutions followed by biotinylated goat anti-mouse IgG2b (Seralab Ltd, Sussex), and finally a mixture of streptavidin Texas red (1 in 100) and FITC conjugated goat anti-mouse IgG1 (Southern Biotechnology Associates, via Laboratory Impex).

Sections were mounted with Citofluor, a glycerol aqueous mountant, and examined using a Zeiss fluorescence microscope and selective filters.

Significance was determined by the Wilcoxon Rank sum test.

\section{Results}

The numbers of ELAM-1 and VCAM-1 positive cells in each group are shown in figs 1 and 2.

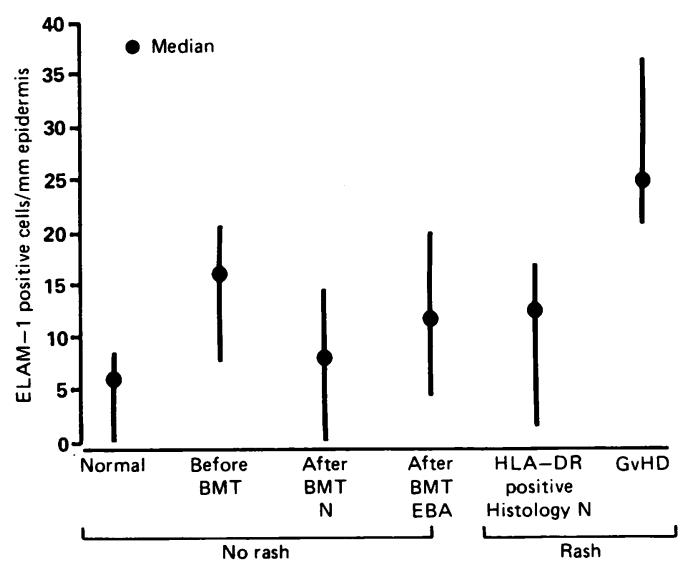

Figure 1 Comparison of number of endothelial cells positive for ELAM-1 in normal donors and recipients of allogeneic marrow before and after transplantation. Results shown as median values with absolute ranges. $N=$ normal $; E B A=$ epidermal basal abnormalities $N=$ normal; $E B A=$ epidermal basal abnormalities
without lymphocytic infiltration; $G v H D=$ graft-versushost disease. 
Figure 2 Comparison of number of perivascular and dermal dendritic cells positive for VCAM-1 in normal donors and recipients of allogeneic marrow before and after transplantation. Result shown as median values with absolute ranges.

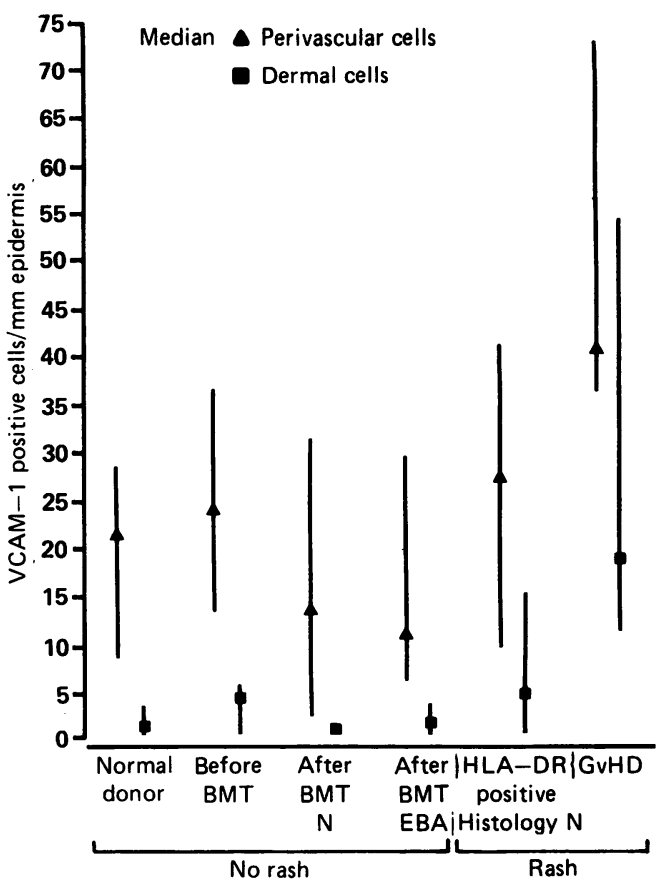

NORMAL DONORS AND BIOPSY SPECIMENS BEFORE TRANSPLANTATION

Each control biopsy specimen was histologically normal and exhibited HLA-DR negative epidermal keratinocytes. Immunostaining for ELAM-1 was confined to vascular endothelial cells (fig 3A). VCAM-1 positivity was seen on perivascular dendritic cells with long fine processes completely encircling the vascular endothelium (fig 4). In several specimens phagocytosed melanin was seen in these perivascular cells (fig 5). Similar dendritic cells were also seen around hair follicles, sweat glands, and ducts and in very small numbers dispersed in the papillary dermis.

The median values and ranges for ELAM$1+$ and VCAM $-1+$ cells are shown in figs 1 and 2 .

\section{BIOPSY SPECIMENS FROM PATIENTS WITHOUT} RASHES AFTER TRANSPLANTATION

Of the 10 biopsy specimens, four showed normal histology and six EBA. All were negative for HLA-DR on keratinocytes. The distribution of ELAM-1 and VCAM-1 staining was similar to that seen in normal donors and biopsy specimens before transplantation. Median values and ranges are summarised in figs 1 and 2 .

BIOPSY SPECIMENS FROM PATIENTS WITH RASHES AFTER TRANSPLANTATION

Of the 15 biopsy specimens taken from sites of a macular, erythematous rash, seven fulfilled the diagnostic histological criteria for GvHD, having epidermal basal cell vacuolation and degeneration, individual cell necrosis of keratinocytes, and a lichenoid lymphocytic infiltrate in the upper dermis and lower epidermis. These specimens showed positive immunostaining for HLA-DR antigens on epidermal keratinocytes.

The remaining eight lacked the diagnostic lymphocytic infiltrate, histologically, six showing EBA and two normal histology, but they did show keratinocyte HLA-DR positivity. Median values and ranges for ELAM-1 and VCAM-1 positive cells are shown in figs 1 and 2. ELAM-1 positive cells were restricted to dermal vessels and appeared as cords of uniformly stained endothelial cells (fig 3B). Their number was significantly increased in the seven biopsy specimens exhibiting histological evidence of GvHD when compared with those from normal donors, patients before transplantation, transplant recipients exhibiting normal histology or EBA, and patients with rashes exhibiting HLA-DR keratinocyte positivity $(\mathrm{p}=<0.02)$.

VCAM-1 positive cells were seen in GvHD not only around vessels and accessory structures but also as dendritic cells freely dispersed throughout the upper dermis (fig 6). The perivascular and free dermal cells were increased in GvHD compared with biopsy specimens from normal donors, patients before transplantation, transplant recipients exhibiting normal histology or EBA, and biopsy specimens with HLA-DR positive keratinocytes but without unequivocal GvHD histology $(\mathrm{p}=<0.02)$.

A comparison of specimens taken from this last group of patients with those taken after transplantation with no evidence of rash (EBA or normal histology), showed that there was no significant increase in the number of ELAM-1 positive endothelial cells but there was, however, a significant increase in VCAM-1 positive cells around vessels $(p=0.02)$ and dispersed free in the dermis $(p=0.05)$.

DOUBLE LABELLING

All ELAM-1 positive cells were also positive with factor VIII related antigen by double label immunofluorescence studies, confirming that ELAM-1 staining was restricted to endothelial cells.

VCAM-1 positive cells were also positive by double labelling with Leuk 19. Studies combining Leuk 19 with EBM11 (CD68) showed all Leuk $19+$ cells to be EBM11+. Consequently, all VCAM-1 + cells were CD68 + . VCAM-1 + cells were generally negative on double labelling with factor VIII related antigen and completely negative on double labelling with anti-CD3 antibody, showing that VCAM-1 positivity was rarely seen on endothelial cells and never on perivascular $T$ lymphocytes.

\section{Discussion}

In this study we have shown an increase in two vessel associated cell adhesion molecules, ELAM-1 and VCAM-1, in acute cutaneous GvHD. This follows a similar study in which we showed increases in a third adhesion molecule, ICAM-1. ${ }^{6}$ Each of these three molecules shows a distinctive pattern of expression in the normal skin and in GvHD. In normal controls, ICAM-1 is present mainly on dermal leucocytes, endothelial cells, and a few keratinocytes, ELAM-1 weakly on endothelial 


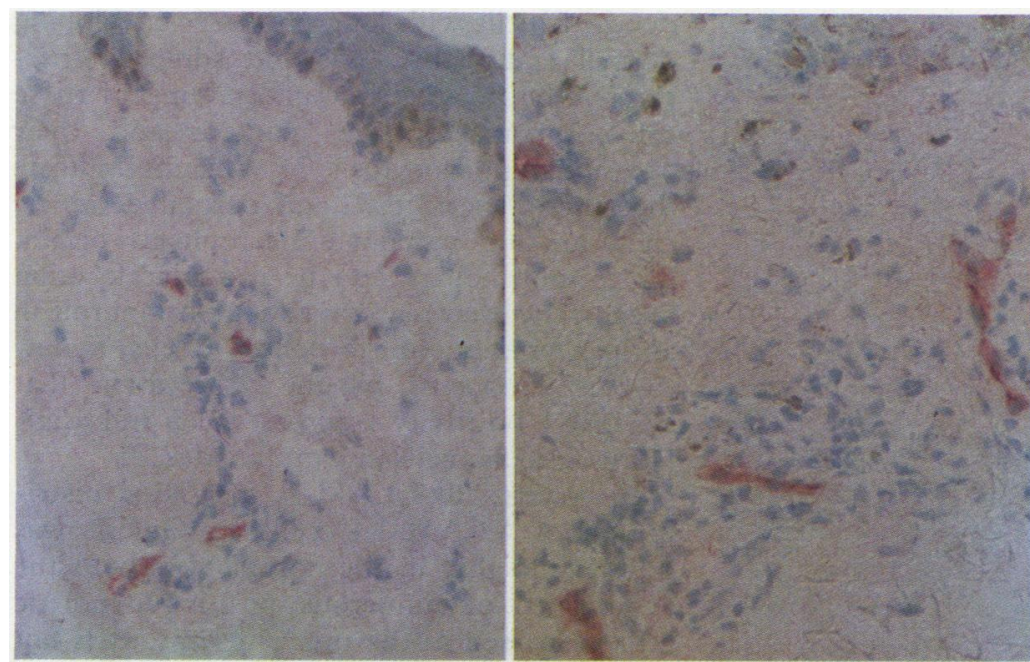

Figure 3 (a) ELAM-1 staining of a biopsy specimen from a normal donor showing weak positivity of a small number of vascular endothelial cells.

(b) ELAM-1 staining of a transplantation biopsy specimen showing GvHD. There is strong positivity of cords of uniformly stained vascular endothelial cells.

cells only, and VCAM-1 on dendritic cells mainly surrounding blood vessels and adnexal structures. In GvHD there is a demonstrable increase in ICAM-1 on keratinocytes, ELAM-1 on endothelial cells, and VCAM-1 on dendritic cells both around the vessels and dispersed in the upper dermis.

A major reason for undertaking the present study was to look for markers of early GvHD as we have found conventional histological examination to be a relatively ineffective method of diagnosing the disorder in its initial stages. ${ }^{3}$ Vessel associated molecules seemed an appropriate choice as the cells of the vascular complex are those first encountered by donor leucocytes entering the skin.

Furthermore, there is some clinical and experimental evidence of involvement of vessels in GvHD. Dumler et al reported lymphocytic infiltration of the intima of dermal blood vessels, perivascular nuclear dust deposition and extravasation of factor VIII related antigen. ${ }^{10}$ Snover et al observed "endothelialitis" in hepatic GvHD," and

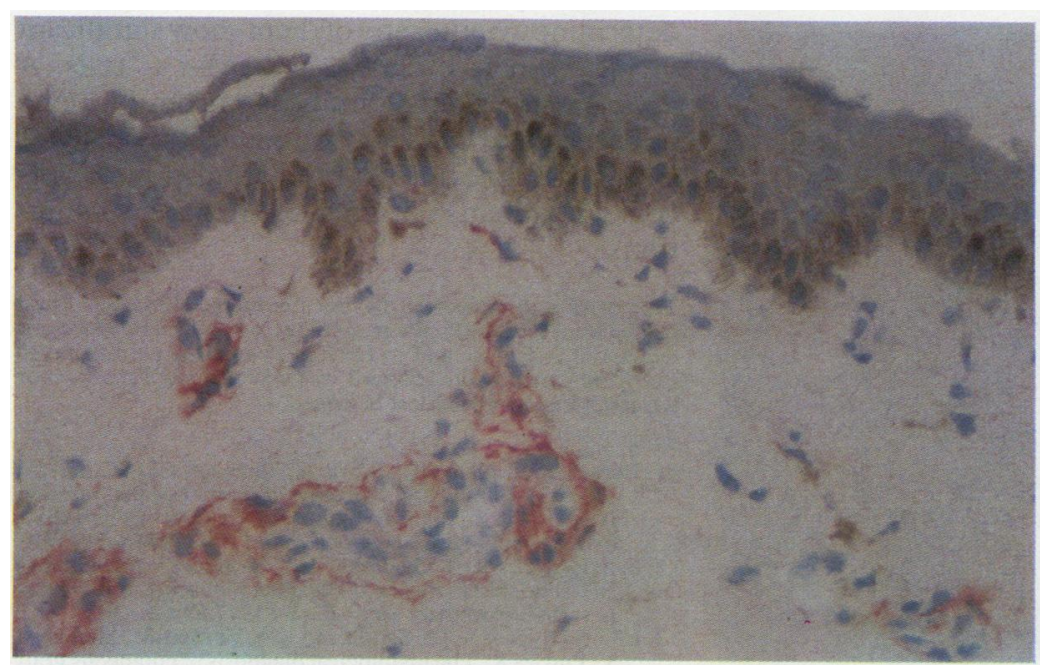

Figure 4 VCAM-1 staining of a biopsy specimen from a normal donor showing positivity of perivascular dendritic cells with long fine processes encircling the vascular
endothelium.
Nonomura et al showed increased lymphocyte adhesion to hepatic vascular endothelium after bone marrow transplantation which paralleled the severity of portal tract infiltrates. ${ }^{12}$ Lymphocytes from rats with early acute GvHD have been reported to show antigen specific injury to cultured endothelial cells. ${ }^{13}$

Lymphocytic infiltration into tissues is preceded by their adhesion to vascular endothelium. This lymphocyte endothelial interaction is regulated by various adhesion molecules whose expression may be modified by local cytokine release. ${ }^{14}$

ELAM-1 is a member of the selectin family of adhesion molecules. Its expression is increased temporarily on cultured endothelial cells by the addition of IL- $1 \alpha$, IL- $\beta$, or tumour necrosis factor, with peak expression at four to six hours, diminishing noticeably at 24 hours. In human skin in vivo, however, the time sequence for ELAM-1 expression has been shown to be longer and may persist for days after the onset of inflammation. ${ }^{1516}$

In normal skin we found ELAM-1 positivity on a variable and generally small number of endothelial cells with significant increases after transplantation only when there was unequivocal histological evidence of GvHD. No increase was seen in patients with only clinical evidence of the disease who lacked demonstrable cutaneous leucocytic infiltration, even in the presence of increased keratinocyte HLADR staining. The association between leucocytic infiltration and endothelial ELAM-1 expression in GvHD is not entirely clear from the present study. It is possible, however, that increases in ELAM-1 precede leucocytic infiltration and are important in allowing mononuclear cells to pass from the blood to the dermis. Our failure to show endothelial ELAM-1 positivity without (and presumably before) leucocytic infiltration could be explained by a short time interval between the two events, or the relative insensitivity of immunohistology in detecting small but pathologically important increases in the adhesion molecule. Alternatively, mononuclear cell traffic into the dermis at early time points in the evolution of GvHD may not be critically dependent on this adhesion molecule.

Even in GvHD, not all endothelial cells exhibited ELAM-1 positivity, but a lack of suitable markers prevented us from determining the precise nature of the positive and negative cells. The morphological features of the positive vessels suggested that they were blood rather than lymphatic in type. The continuous, rather than patchy, positivity in GvHD would be in keeping with a functional zone of dermal vessels concerned with regulating leucocyte ingress. ${ }^{17}$

VCAM-1 is a member of the immunoglobulin super gene family which selectively binds to VLA-4 (CD49D/CD29), a $\beta$-1 integrin expressed on lymphocytes, monocytes, and neural crest derived cells. ${ }^{818}$ It is detectable on the surface of cultured endothelial cells three hours after stimulation by TNF with peak expression at six to 10 hours, and persists for at least 72 hours in the presence 
Figure 5 VCAM-1 positive perivascular dendritic cells containing phagocytosed melanin pigment.

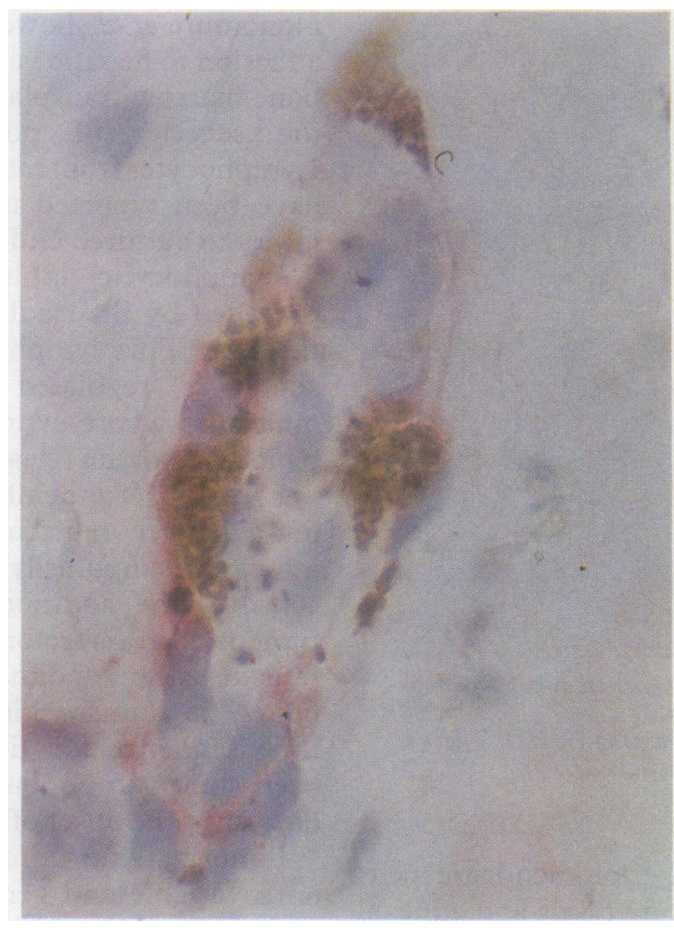

of cytokine, a prolonged time course consistent with lymphocyte and monocyte migration in chronic inflammatory lesions seen in vivo. ${ }^{19} \mathrm{~A}$ more transient expression is seen after stimulation by IL- 1 and lipopolysaccharide. ${ }^{9}$ Although 1.4C3 and other monoclonal antibodies against VCAM-1 have been shown to bind to endothelial cells in culture, and venules in tissue sections of cutaneous delayed hypersensitivity reactions to tuberculin and insect bite, ${ }^{1620}$ we observed very little staining of cutaneous endothelial cells in the present study either in normal controls or in cases of GvHD. The differences in adhesion molecule expression in GvHD compared with delayed hypersensitivity may reflect differences in the cytokines involved.

There was strong VCAM-1 positivity of

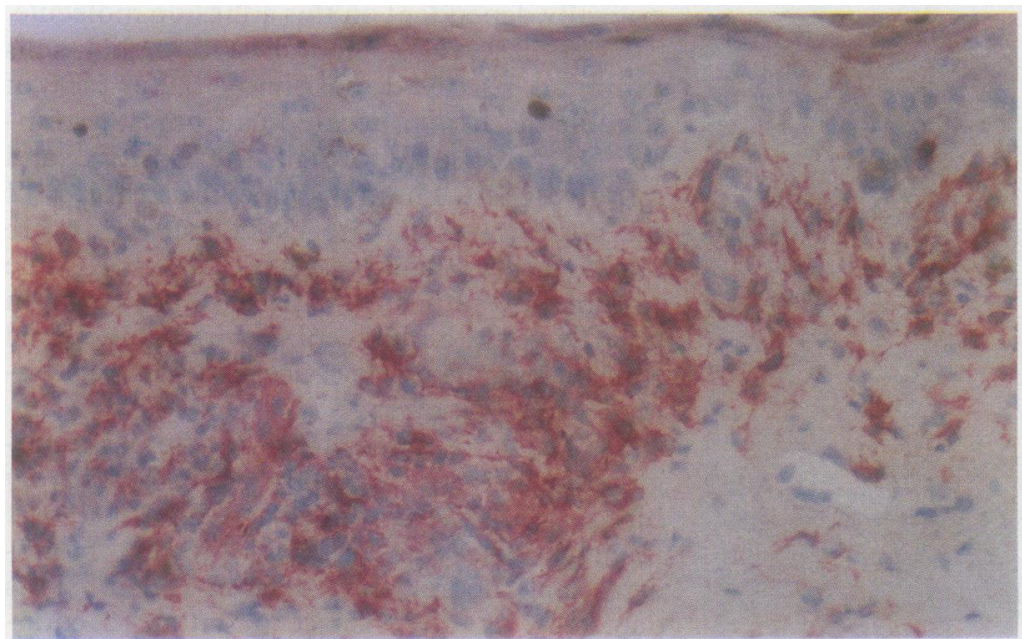

Figure 6 VCAM-1 staining of a transplantation biopsy specimen showing GvHD. There is strong positivity of perivascular cells together with dendritic cells dispersed throughout the upper dermis. dendritic cells with long processes enveloping dermal endothelial cells and adnexal structures. The positive immunostaining of these cells for macrophage markers and their occasional phagocytosis of melanin indicate that these "pericytes" and dermal dendritic cells are of monocyte/macrophage origin. Their morphology and perivascular location suggest that they may have an important role in antigen presentation to donor $\mathrm{T}$ cells, a view that is supported by the observation that VCAM-1 positivity is also seen on interdigitating reticular cells in lymph nodes. ${ }^{920}$

In contrast to ELAM-1, the tissue distribution of VCAM-1 + cells in GvHD was different to that in the normal skin with the appearance of numerous positive dendritic cells dispersed in the upper dermis. It is not clear at present to what extent the appearance of these cells is due to infiltration or a change in phenotype of existing cells. The latter mechanism must operate to some extent as there was a greater increase in VCAM-1 + cells than Leuk $19+$ cells and the latter included all the former. Interactions between the VCAM-1 + dermal "dendrocytes" and donor T cells could be important in the pathogenesis of tissue damage in acute cutaneous GvHD and, as suggested above for VCAM-1 + perivascular cells, could involve antigen presentation. In this context it would be interesting to determine the rate at which recipient cells of each of these types are replaced by those of donor origin. Further studies are under way to investigate this.

The increase in VCAM-1 positive cells in biopsy specimens without detectable lymphocytic infiltration, taken soon after the onset of rash, raises the possibility of a role for this antibody in diagnosing early GvHD. Enumerating increases in perivascular cells would be time-consuming and largely meaningless unless counts before transplantation were available, but positive cells dispersed free in the dermis would be much easier to evaluate as they were almost entirely undetectable in the control biopsy specimens from transplant recipients. As some patients with early GvHD lack lymphocytic infiltration and keratinocyte HLA-DR positivity ${ }^{4}$ it would be of interest to determine whether any of them show significant increases in free dermal VCAM-1 + cells. A detailed prospective study is currently being undertaken to evaluate antibodies to VCAM-1 and other molecules in predicting clinical outcome in patients with rashes after transplantation. This work was supported by the Leukaemia Research Fund
(LRF Grant No 90/01). We are grateful to Mrs Valerie Power for (LRF Grant No 90/01). We are grateful to Mrs Valerie Power for
secretarial assistance and to Dr Ray Powles for permission to study his patients. Dr Haskard was a Wellcome Trust Senior Research Fellow in Clinical Science.

1 Lerner KG, Kao GF, Storb R, Buckner CD, Clift RA, Thomas ED. Histopathology of graft-vs-host reaction (GvHR) in human recipients of marrow from HLAmatched sibling donors. Transplant Proc 1974;6:367-71.

2 Sale GE, Lerner KG, Barker EA, Shulman HM, Thomas ED. The skin biopsy in the diagnosis of acute graft versus host disease in man. Am J Pathol 1977;89:621-36.

3 Elliott CJ, Sloane JP, Sanderson KV, Vincent M, Shepherd $V$, Powles $R$. The histological diagnosis of cutaneous graft versus host disease: relationship of skin changes to marrow purging and other clinical variables. Histopathology 1987;11:145-55.

4 Sloane JP, Elliott CJ, Powles R. HLA-DR expression in 
epidermal keratinocytes after allogeneic bone marrow transplantation. Transplantation 1988;46:840-4.

5 Griffiths CE, Voorhees JJ, Nickoloff BJ. Characterisation of intercellular adhesion molecule-1 and HLA-DR expression in normal and inflamed skin: modulation by recombinant gamma interferon and tumour necrosis factor. J Am Acad Dermatol 1989;20:617-29.

6 Norton J, Sloane JP. ICAM-1 expression on epidermal keratinocytes in cutaneous graft-versus-host disease. Transplantation 1991 (in press).

7 Bevilacqua MP, Stengelin S, Gimbrone MA, Seed B. Endothelial leukocyte adhesion molecule 1: an inducible Endothelial leukocyte adhesion molecule 1 : an inducible
receptor for neutrophils related to complement regulatory receptor for neutrophils related to complement
proteins and lectins. Science $1989 ; 243: 1160-4$.

proteins and lectins. Science 1989;243:1160-4.
8 Osborn L, Hession C. Tizard R, et al. Direct expression cloning of vascular cell adhesion molecule 1 (VCAM1) a cytokine-induced endothelial protein that binds to lymphocytes. Cell 1989:59:1203-11.

9 Wellicome SM, Thornhill MH, Pitzalis C, et al. A monoclonal antibody that detects a novel antigen on endothelial cells that is induced by tumour necrosis factor, IL-1, or lipopolysaccharide. J Immunol 1990;144:2558-65.

10 Dumler JS, Beschorner WE, Farmer ER, Di Gennaro KA, Saral R, Santos GW. Endothelial cell injury in cutaneous acute graft-versus-host disease. Am J Pathol 1989; 135:1097-103.

11 Snover DC, Weisdorf SA, Ramsay NK, McGlave P, Kersey JH. Hepatic graft versus host disease: a study of the predictive value of liver biopsy in diagnosis. Hepatology 1984;4:123-30.

12 Nonomura A, Kono N, Yoshida K, Mizukami Y, Matsubara F, Nakanuma Y. Histological changes of the liver in experimental graft-versus-host disease across minor histocompatibility barriers. IV. A study of lymphocytehistocompatibility barriers. IV. A study of lymphocyte13 Beschorner WE, Shinn CA, Hess AD, Suresch DL, Santos GW. Immune-related injury to endothelium associated with acute graft-versus-host disease in the rat. Transplant Proc 1989;21:3025-7.

14 Springer TA. Adhesion receptors of the immune system. Nature 1990;346:425-34.

15 Pober JS. Cytokine-mediated activation of vascular endothelium. Am J Pathol 1988;133:426-33.

16 Norris P, Poston RN, Thomas DS, Thornhill M, Hawk J, Haskard DO. The expression of endothelial leukocyte adhesion molecule-1 (ELAM-1), intercellular adhesion molecule-1 (ICAM-1) and vascular cell adhesion molecule-1 (VCAM-1) in experimental cutaneous inflamdelayed hypersensitivity. J Invest Dermatol 1991 (in press).

17 Messadi DV, Pober JS, Fiers W, Gimbrone MA, Murphy GF. Induction of an activation antigen on postcapillary venular endothelium in human skin organ culture. $J$ Immunol 1987;139:1557-62.

18 Elices MJ, Osborn L, Takada Y, et al. VCAM-1 on activated endothelium interacts with the leukocyte integrin VLA-4 at a site distinct from the VLA-4/fibronectin binding site. Cell 1990;60:577-84.

19 Osborn L. Leucocyte adhesion to endothelium in inflammation. Cell 1990;62:3-6.

20 Rice GE, Munro JM, Bevilacqua MP. Inducible cell adhesion molecule 110 (INCAM-110) is an endothelial receptor for lymphocytes. $J$ Exp Med 1990;171:1369-74. 